\title{
Stop Flow Lithography Synthesis and Characterization of Structured Microparticles
}

\author{
David Baah, ${ }^{1}$ Tobias Donnell, ${ }^{2}$ Sesha Srinivasan, ${ }^{3}$ and Tamara Floyd-Smith ${ }^{1,2}$ \\ ${ }^{1}$ Materials Science \& Engineering Department, Tuskegee University, Tuskegee, AL 36088, USA \\ ${ }^{2}$ Chemical Engineering Department, Tuskegee University, Tuskegee, AL 36088, USA \\ ${ }^{3}$ College of Innovation and Technology, Florida Polytechnic University, Lakeland, FL 33805, USA \\ Correspondence should be addressed to Tamara Floyd-Smith; tfloyd@mytu.tuskegee.edu
}

Received 8 August 2014; Revised 10 November 2014; Accepted 11 November 2014; Published 31 December 2014

Academic Editor: Theodorian Borca-Tasciuc

Copyright (C) 2014 David Baah et al. This is an open access article distributed under the Creative Commons Attribution License, which permits unrestricted use, distribution, and reproduction in any medium, provided the original work is properly cited.

\begin{abstract}
In this study, the synthesis of nonspherical composite particles of poly(ethylene glycol) diacrylate (PEG-DA)/SiO ${ }_{2}$ and PEG$\mathrm{DA} / \mathrm{Al}_{2} \mathrm{O}_{3}$ with single or multiple vias and the corresponding inorganic particles of $\mathrm{SiO}_{2}$ and $\mathrm{Al}_{2} \mathrm{O}_{3}$ synthesized using the Stop Flow Lithography (SFL) method is reported. Precursor suspensions of PEG-DA, 2-hydroxy-2-methylpropiophenone, and $\mathrm{SiO}_{2}$ or $\mathrm{Al}_{2} \mathrm{O}_{3}$ nanoparticles were prepared. The precursor suspension flows through a microfluidic device mounted on an upright microscope and is polymerized in an automated process. A patterned photomask with transparent geometric features masks UV light to synthesize the particles. Composite particles with vias were synthesized and corresponding inorganic $\mathrm{SiO}_{2}$ and $\mathrm{Al}_{2} \mathrm{O}_{3}$ particles were obtained through polymer burn-off and sintering of the composites. The synthesis of porous inorganic particles of $\mathrm{SiO}_{2}$ and $\mathrm{Al}_{2} \mathrm{O}_{3}$ with vias and overall dimensions in the range of $\sim 35-90 \mu \mathrm{m}$ was achieved. BET specific surface area measurements for single via inorganic particles were $56-69 \mathrm{~m}^{2} / \mathrm{g}$ for $\mathrm{SiO}_{2}$ particles and $73-81 \mathrm{~m}^{2} / \mathrm{g}$ for $\mathrm{Al}_{2} \mathrm{O}_{3}$ particles. Surface areas as high as $114 \mathrm{~m}^{2} / \mathrm{g}$ were measured for multivia cubic $\mathrm{SiO}_{2}$ particles. The findings suggest that, with optimization, the particles should have applications in areas where high surface area is important such as catalysis and sieving.
\end{abstract}

\section{Introduction}

Organic and inorganic micro- and nanoparticles and their composites with tailored morphologies exhibit unique structural or shape dependent phenomena. For these particles, control of shape and morphology are desirable for improved application performance. For example, nonspherical particles are promising as drug delivery vehicles due to the dramatic increase in surface area-to-volume ratio (as a result of departure from spherical shape to nonspherical shape) and improved surface interaction (due to larger surface area) leading to increased cellular uptake into target organs $[1$, 2]. Consequently, efforts to develop new methods (both "bottom-up" and "top-down") to synthesize particles with unique structural features have intensified. Recent reviews of the literature highlight developments toward shape control of micro- and nanoparticles with focus on scientifically and technologically important particles [3, 4]. Burda et al. (2005) explained that, with the bottom-up approach, particle formation begins with nucleation and homogenous nucleation is governed by the driving forces of thermodynamics [5]. For spherical particles, this driving force is expressed by the free energy change $(\Delta G)$ of particle formation expressed in

$$
\Delta G(r)=-\frac{4}{V} \pi r^{3} k_{B} T \operatorname{In}(S)+4 \pi r^{2} \gamma,
$$

where $V$ is the molecular volume of the precipitated species, $r$ is the radius of the nuclei, $k_{B}$ is the Boltzmann constant, $S$ is the saturation ratio, and $\gamma$ is the surface energy per unit surface area [5]. Even though it is the contention of the authors that particle growth follows the path of minimum energy which largely favors the formation of spherical particles, they posited that particle formation is kinetically driven and that any metastable shape can be arrested by changing the reaction conditions. Consequently, surfactant molecules and different concentrations of monomers as capping agents, as well as 
varying temperatures and $\mathrm{pH}$ of precursor solutions, are used as shape, size, and morphological control parameters following nucleation and during particle growth [5-9]. Conditions, such as slowing down the rate of polycondensation reaction in the sol gel synthesis of $\mathrm{SiO}_{2}$ nanoparticles, yield smaller sized particles whereas larger sized particles are obtained by increasing the ammonia concentration [10]. Other authors have discussed the use of emulsion droplets, hard and soft templates, sacrificial templates, and template free approaches for the synthesis of particles with unconventional structures and their applications in batteries, biomedicine, catalysis, and sensing [11-13]. Additionally, the independent or combined applications of the principles of Oswald ripening and Kirkendall effects have been used to achieve the synthesis of generally spherical and pseudospherical particles with hollow cavities for applications in gas adsorption and energy storage [14-22]. Like hollow particles, the introduction of small vias or ordered macropores is a novel means of creating structures with increased surface area [23].

Given the potential of nonspherical particles, new approaches have been developed to provide additional methods to fill the need to synthesize them. While microfluidics technology is diverse in its usage including evaluation of nanobiomaterials and controlled release [24, 25], its application in particle design and synthesis is unparalleled. Recent articles have catalogued specific microfluidic platforms, particle formation precursors, and conditions which produce myriad particles with unique morphological features and production rates [26-28]. For example, the stop flow lithography (SFL) technique, used in this study, produces particles at a rate of $0.2 \mathrm{~g} / \mathrm{hr}$ and depends on the free radical polymerization of the precursor onto which UV light is projected using a microscope. SFL is limited to extrusion of two-dimensional particles and, therefore, is an excellent method for synthesis of nonspherical particles for the current and potential applications that benefit from nonspherical particle shapes. SFL controls the particle axial dimension and the cross-sectional area using the microchannel depth and objective lens magnification, respectively. The versatility of this method extends to the ability to synthesize encoded particles for microRNA detection [29] with dimensions as small as $800 \mathrm{~nm}$ realized [30]. In previous work by the author and others, the SFL technique has been used to demonstrate the synthesis of nonspherical organic particles and their composites [31-33] as well as metal and metal oxide particles [34-36]. However, niche application requirements of metal oxide particles demand particles with ordered porosity which possess important physical properties, such as high surface area, favorable to heterogeneous catalysis, supports, sieves, and adsorbents [37, 38].

In this paper, we report the synthesis of two-dimensionally extruded microparticles with single and multiple vias and different cross-sectional shapes using the SFL method. The particles reported in this paper consist of high fidelity and solvent free PEG-DA/SiO 2 and PEG-DA/ $\mathrm{Al}_{2} \mathrm{O}_{3}$ composite particles and their corresponding inorganic particles obtained through binder burn-out. In this work, microstructured particles are those in which vias are introduced with the aim of imparting additional surface area. The corresponding particle nanostructure results from the inorganic nanoparticles used to synthesize the microparticles. This paper is significant because it extends our work on the synthesis of solid metal oxide particles using SFL to microstructured particles with potential applications in the areas of catalysis, supports, sieves, and adsorbents. Also, to our knowledge, it provides the first detailed characterization of the nanostructure of these types of particles. $\mathrm{SiO}_{2}$ and $\mathrm{Al}_{2} \mathrm{O}_{3}$ are common catalyst support materials and were chosen because $\mathrm{SiO}_{2}$ has a low melting point and $\mathrm{Al}_{2} \mathrm{O}_{3}$ was simultaneously being investigated for abrasives applications. However, in theory, this method is applicable to any metal oxide or other material that can be acquired in nanoparticle form and has a melting point higher than $600^{\circ} \mathrm{C}$, the temperature above which all polymer binder is removed.

\section{Experimental}

2.1. Materials. Precursor suspensions are prepared using a commercial $\mathrm{SiO}_{2}$ dispersion in water (Ludox AS-40 or Ludox CL-X colloidal silica), poly(ethylene glycol) diacrylate, 2hydroxy-2-methylpropiophenone, hydrogen peroxide, and ascorbic acid all supplied by Sigma Aldrich, as well as an aluminum oxide nanodispersion $\left(\mathrm{Al}_{2} \mathrm{O}_{3}, 20 \mathrm{wt} \%, 30 \mathrm{~nm}\right)$ supplied by US Research Nanomaterials Inc. The particle synthesis was conducted using geometrically patterned photomasks and in microfluidic devices whose fabrication methods were described in a preceding work [31, 39].

2.2. Particle Synthesis and Collection. Figure 1 is a schematic illustration of the particle synthesis workstation. Patterned photomasks mask the UV light from a source. This photomask is inserted in the field stop of an upright microscope (Olympus BX51WF Microscope) and changed, when necessary, to change the imposed cross-sectional shapes of the particles. The UV light is focused onto the precursor flow stream using a $10 x$ or $20 x$ microscope objective lens. In addition to light focusing, the objective controls the particle cross-sectional size with the particle size decreasing as the objective magnification increases. The axial dimension of the particles is controlled by the microfluidic channel depth. In a typical operation, a three-way solenoid valve regulates the airpressure-driven precursor solution supply to the microfluidic device mounted on the stage of a microscope. The precursor is prepared by mixing aliquots of the commercial suspensions of the inorganic nanoparticles with PEG-DA to constitute suspensions containing $10 \mathrm{wt} \% \mathrm{Al}_{2} \mathrm{O}_{3}$ and $20 \mathrm{wt} \% \mathrm{SiO}_{2}$ in $30 \mathrm{wt} \%$ PEG-DA. The goal was to use the highest possible concentration of nanoparticles in the suspension, but the concentration was limited by excessive fluid resistance to flow (high viscosity) caused by the particle loading as well as light scattering and consequent inadequate binder crosslinking in spite of index matching. The source of precursor supply is a $10 \mathrm{~mL}$ syringe, with the tip connected to the microfluidic device through ethyl vinyl acetate microbore tubing of $0.04^{\prime \prime}$ ID (WU-06492-04 Cole Parmer). The light source is a Lumen 200 (Prior Scientific) equipped with a 200 watt metal arc bulb connected to a Shutter Driver (VCM-D1, Uniblitz) whose opening and closure are synchronized with those of 


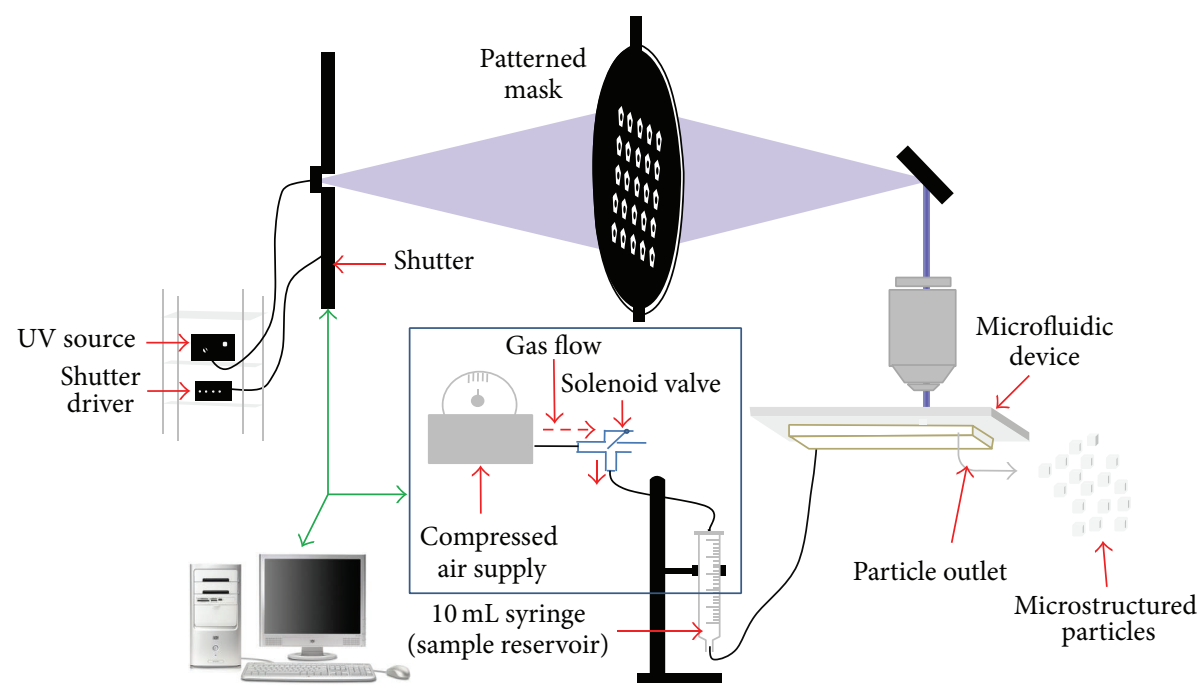

FIGURE 1: Schematic of the stop flow lithography (SFL) setup consisting of a UV light source, a microfluidic device mounted on an upright microscope, and a prepolymer supply reservoir mounted on a clamp stand and connected to a solenoid valve to regulate sample flow to the device and particle ejection from the device.

the solenoid valve to control the introduction of fresh precursor solution as well as expelling synthesized particles. A $365 \mathrm{~nm}$ bright-line filter (Max Lamp Mercury Line FilterHg01-365-25; Semrock) is positioned in the light path to select the appropriate wavelength of $365 \mathrm{~nm}$ for the photoactivation. Once the shutter is opened, the light comes through to the sample and a free radical polymerization is triggered which lasts for the period the light is on. Typically with this method, particles are synthesized in 75-350 ms exposure times in a fully automated process $[36,39,40]$.

Particle separation is by gravity settling. The process is described in an earlier report [31]. Further, wash reagents (10 vol\% hydrogen peroxide and $15 \mathrm{wt} / \mathrm{vol} \%$ ascorbic acid) are used for final washing to maintain particle fidelity. Once the composite particles are collected, washed free from unreacted precursor, and stabilized, they are sintered using a $1500^{\circ} \mathrm{C}$ Compact Muffle Furnace (KSL-1500X-S) equipped with a programmable controller. For the composite $\mathrm{SiO}_{2}$ particles, the furnace is ramped from room temperature to $600^{\circ} \mathrm{C}$ at $5^{\circ} \mathrm{C} / \mathrm{min}$ to first remove moisture and then burn off the polymer binder. The temperature is held at $600^{\circ} \mathrm{C}$ for $30 \mathrm{~min}$ to ensure complete removal of binder and then ramped again at $\sim 4.5^{\circ} \mathrm{C} / \mathrm{min}$ to $1150^{\circ} \mathrm{C}$ and held for 8 hours. Finally, controlled cooling is conducted at $\sim 3^{\circ} \mathrm{C} / \mathrm{min}$ to $100^{\circ} \mathrm{C}$ and the furnace shuts off while the particles continue to cool to room temperature. The process described produces consolidated ceramic particles. Sintering of $\mathrm{PEG}-\mathrm{DA} / \mathrm{Al}_{2} \mathrm{O}_{3}$ composite particles follows a similar profile except that the maximum temperature is $1450^{\circ} \mathrm{C}$, near the limit of the equipment, with at least a two-hour hold time.

2.3. Characterization. The zeta potential of the particles in suspension was measured using a Zeta Potential Analyzer (ZetaPlus, BIC). First, aliquots of the mother liquor are centrifuged at $5000 \mathrm{rpm}$ for 10 mins and the supernatant was collected. A few drops of the mother liquor are added to
TABLE 1: Zeta potential of $\mathrm{SiO}_{2}$ and $\mathrm{Al}_{2} \mathrm{O}_{3}$ nanoparticles.

\begin{tabular}{lcc}
\hline & $\mathrm{SiO}_{2}$ & $\mathrm{Al}_{2} \mathrm{O}_{3}$ \\
\hline Solvent & Ludox/Water $(n=10)$ & Isopropanol $(n=10)$ \\
Concentration (\% wt) & 40 & 20 \\
Particle size $(\mathrm{nm})$ & 15 & 30 \\
Zeta potential $(\mathrm{mV})$ & $-20.9 \pm 3.9$ & $36.0 \pm 2.6$ \\
\hline
\end{tabular}

the supernatant, mixed thoroughly, and used as sample. Prior to sample measurement, a zeta potential reference material (BI-ZR3) is analyzed to check the instrument performance and reliability.

Scanning electron microscopic (SEM) images of the samples were collected to show their microstructural features. First, approximately $5 \mathrm{mg}$ of the dried aliquot sample is placed on a conductive carbon tape attached to the top of a $13 \mathrm{~mm}$ radius aluminum stub and placed in an EMS 550X Sputter Coating machine to coat the sample surface in a thin film of gold. Subsequently, the sample is imaged using a JOEL JSM 5800 or ZEISS EVO 50VP scanning electron microscope.

The surface area and pore size distribution analyses are carried out using Quantachrome Instruments' Autosorb-iQ BET surface area analysis. Approximately $0.5 \mathrm{~g}$ of sample is loaded in a $6 \mathrm{~mm}$ tube with a filler rod and outgassed for at least $3 \mathrm{hrs}$ at $300^{\circ} \mathrm{C}$, after which a 7-point nitrogen physisorption isotherm is performed at the liquid nitrogen temperature of $77 \mathrm{~K}$. The BET (Brunauer-Emmett-Teller) surface area of the samples is determined using $\mathrm{ASiQwin}$ II data analysis software.

\section{Results and Discussion}

Table 1 shows results from the zeta potential characterization of the precursor metal oxide particles. The high absolute 


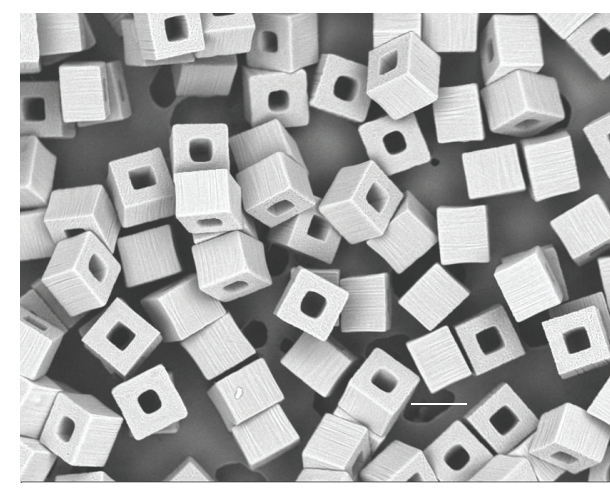

(a)

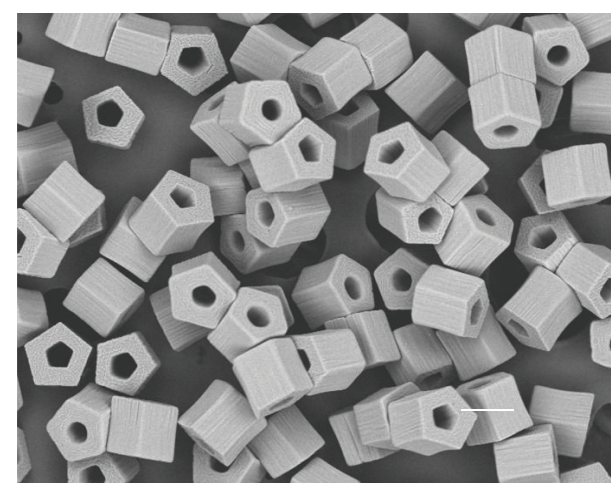

(b)

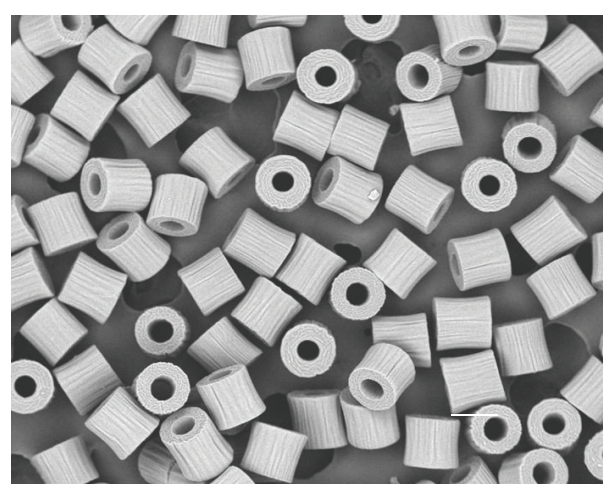

(c)

FIGURE 2: SEM micrographs of solvent free $\mathrm{SiO}_{2} / \mathrm{PEGDA}$ composite particles with single vias in square, pentagonal, and circular cross-sectional shapes. Scale bar $=100 \mu \mathrm{m}$.

values for $\mathrm{Al}_{2} \mathrm{O}_{3}(36.0 \mathrm{mV})$ and $\mathrm{SiO}_{2}(-20.9 \mathrm{mV})$, respectively, are indications of repulsive interparticle electrostatic interaction suggesting that particle agglomeration due to electrostatic attraction is unlikely. Consequently, one can expect to achieve uniform, stable nanoparticle dispersions as precursor suspensions and the corresponding high fidelity metal oxide particles.

Two different microfluidic devices with channel depths in the range of $60-80$ and $220-250 \mu \mathrm{m}$ are used to synthesize particles with the aim of creating particles with different axial dimensions. Additionally, objective lenses (10x and $20 \mathrm{x}$ ) control cross-sectional size. Square, pentagonal, and circular cross-sectional shapes of particles with single vias are synthesized from the corresponding shape patterned in the photomask. Figures 2(a)-2(c) show SEM micrographs of high fidelity, monodisperse, and solvent free $\mathrm{SiO}_{2} /$ PEGDA composite particles in three cross-sectional shapes. The micrographs in Figure 2 demonstrate that not only are high fidelity composite particles synthesized, but the crosssectional shapes and sizes can be manipulated with relative ease. For example, Figure $2(a)$ is a square cross section of particles measuring $90-120 \mu \mathrm{m}$ in axial and $100-110 \mu \mathrm{m}$ in cross-sectional edge dimensions (220-250 $\mu \mathrm{m}$ device, $10 \mathrm{x}$ objective lens). The corresponding reduced form (images not shown) which measures $57-64 \mu \mathrm{m}$ and $53-59 \mu \mathrm{m}$ in axial and cross-sectional edge dimensions is produced using a 60$80 \mu \mathrm{m}$ depth microfluidic device and a 20x objective lens with the same size transparent feature in the mask. Similar results for pentagonal and circular cross sections have been achieved. With the SFL approach, myriad two-dimensional shapes can be extruded to yield particles. Although the particles in Figure 2 have vias in the shape of the overall particles, other shapes can be introduced.

The synthesis of $\mathrm{Al}_{2} \mathrm{O}_{3} / \mathrm{PEGDA}$ composite particles is also demonstrated. Figure 3 shows SEMs of cubic and rodlike composite particles with vias. A limitation of the $\mathrm{Al}_{2} \mathrm{O}_{3}$ /PEGDA composite particle synthesis is the UV light screening due to $\mathrm{Al}_{2} \mathrm{O}_{3}$ as the weight fraction of $\mathrm{Al}_{2} \mathrm{O}_{3}$ nanoparticles increases. However, in up to $10 \mathrm{wt} \%$ fraction of $\mathrm{Al}_{2} \mathrm{O}_{3}$, it is possible to crosslink the precursor for which the images are shown in Figure 3. These $\mathrm{Al}_{2} \mathrm{O}_{3} / \mathrm{PEG}-\mathrm{DA}$ composite particles, measuring $\sim 100-110 \mu \mathrm{m}$ in axial dimensions, are synthesized with a lower concentration of PEG-DA (25 wt\%) in order to accommodate the viscosity of the stock $\mathrm{Al}_{2} \mathrm{O}_{3}$ nanodispersion. The composite particle integrity is illustrated in Figure 4 which shows a uniform wall thickness for the particles with square cross sections. The thickness of the via walls for the square cross sections is estimated to be in the range of $32-35 \mu \mathrm{m}$ for the $10 \mathrm{x}$ objective and $220-250 \mu \mathrm{m}$ device. 

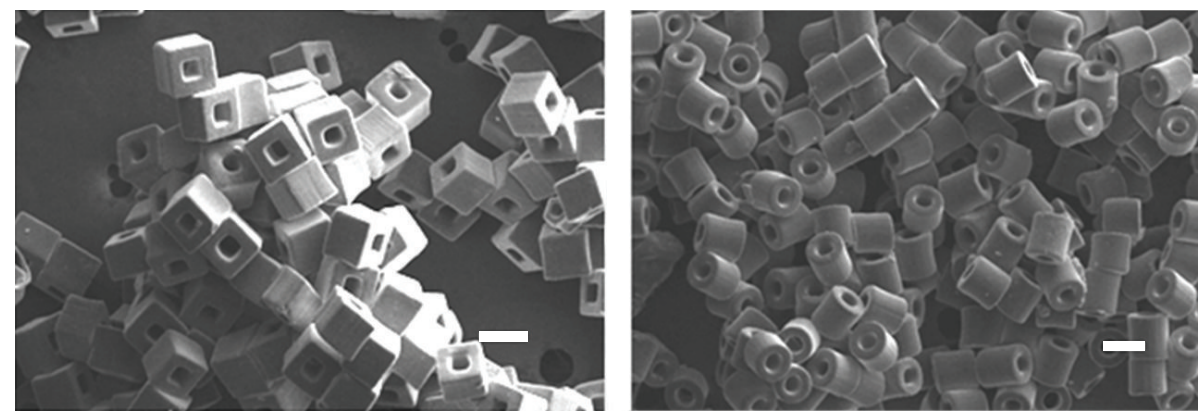

Figure 3: SEM micrographs of solvent free $\mathrm{Al}_{2} \mathrm{O}_{3}$ /PEGDA single via composite particles with square and circular cross-sectional shapes. Scale bar $=100 \mu \mathrm{m}$.

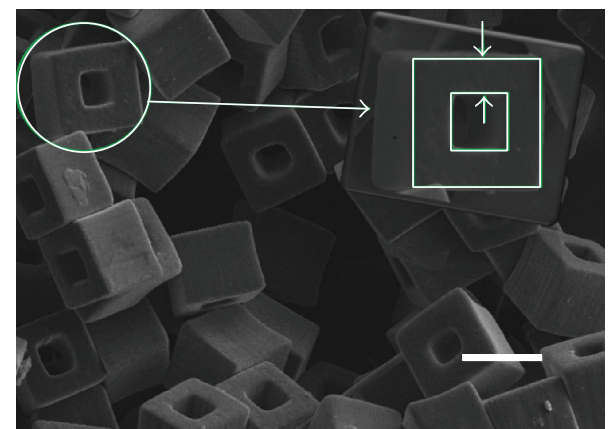

FIGURE 4: Estimation of the wall thickness for the square cross section using the $\mathrm{Al}_{2} \mathrm{O}_{3} / \mathrm{PEG}-\mathrm{DA}$ particles. Scale bar $=100 \mu \mathrm{m}$.

The shape of corresponding inorganic particles was derived from the composites through polymer burn-out and sintering. Figures 5(a)-5(b) are a collection of SEMs showing sintered $\mathrm{SiO}_{2}$ particles with vias in square and pentagonal cross sections. The particle fidelity is further demonstrated in the preservation of particle walls even on sintering the via composite particles. The axial dimensions of the particles for both cross section shapes were in the range of $75-85 \mu \mathrm{m}$ for the $10 \mathrm{x}$ objective and $220-250 \mu \mathrm{m}$ device and $35-45 \mu \mathrm{m}$ for the 20x objective and 60-80 $\mu \mathrm{m}$ device (image not shown).

These particles may have applications for hollow micro/nanomaterials based on properties which include high specific surface areas and inner voids [22]. In many cases, such characteristics make the particles superior to the same sized particle with simple and solid structures. Therefore, they are candidates in many important application areas such as catalysis, sensors [41], Li-ion batteries [12, 15], and drug delivery [42]. It is worth noting that the PEG-DA serves two purposes, (1) as a binder in the composite particles and (2) as a porogenic source in the inorganic particles. Generally, sintering reduces particle porosity. However, the presence of the binder (porogen) is expected to reduce the extent of porosity loss. The high magnification SEM (Figure 6(b)) of the $\mathrm{SiO}_{2}$ particle (inset: Figure 6(a)) surface reveals an agglomerated and tightly packed as well as an uneven particle surface. It appears that the compaction of the composite particles by interparticle electrostatic attractive forces of the constituent inorganic particles is enhanced by the sintering temperature in spite of the departing porogen (polymer binder). It is obvious, from this observation, that the final application of the particles must be considered when choosing the amount of binder, the sintering temperature, the duration of sintering, and other synthesis conditions. The TEM image of the precursor suspension (Figure 6(c)) confirms monodisperse constituent $\mathrm{SiO}_{2}$ nanoparticles which are spherically shaped and approximately $25 \mathrm{~nm}$ in diameter. Our goal is to produce inorganic particles of unique morphologies indirectly by first synthesizing the composites and burning out the binder. Particle size reduction is anticipated as the binder leaves and the inorganic component densifies on heat treatment. For the $\mathrm{SiO}_{2}$ particles, the percent change in the particle volume was calculated and found to range from 58 to $68 \%$ and 47 to $59 \%$ for the $220-250 \mu \mathrm{m}$ and $60-80 \mu \mathrm{m}$ channels, respectively. Assuming that it is applicable to use the bulk density for cristobalite, a volume reduction of $\sim 90 \%$ for a $20 \mathrm{wt} \%$ loading of $\mathrm{SiO}_{2}$ is expected to correspond to a $\sim 50 \%$ reduction in edge length. The difference between the experimental measurements and theoretical predictions is attributed to the porosity of the microparticles and the potential deviation of the nanoparticle density from that of the bulk material. It is also observed from Figure 6(b) that the inorganic component consists of spherically shaped $\mathrm{SiO}_{2}$ particles of approximately $25 \mathrm{~nm}$ diameter which are shown in Figure 6(c).

Figure 7 shows SEM images of $\mathrm{Al}_{2} \mathrm{O}_{3}$ microparticles in circular cross sections imaged in progression of magnification (Figures $7(\mathrm{a})-7(\mathrm{c})$ ) to reveal the microstructural characteristics of the particle surface. The micrographs suggest that the microparticles consist of rod-like $\mathrm{Al}_{2} \mathrm{O}_{3}$ nanoparticles aligned in a pattern. The structural features of $\mathrm{Al}_{2} \mathrm{O}_{3}$ nanoparticles are further revealed in the TEM image of the precursor suspension in Figure 7(d). Also, unlike the $\mathrm{SiO}_{2}$ nanoparticles, the $\mathrm{Al}_{2} \mathrm{O}_{3}$ nanoparticles formed an array with visible interparticle voids (Figure $7(\mathrm{c})$ ). Considering that (1) the weight percent loading of the $\mathrm{Al}_{2} \mathrm{O}_{3}$ composite particles is low and (2) the sintering of $\mathrm{Al}_{2} \mathrm{O}_{3}$ usually requires temperatures in excess of $1600^{\circ} \mathrm{C}$, one would expect 


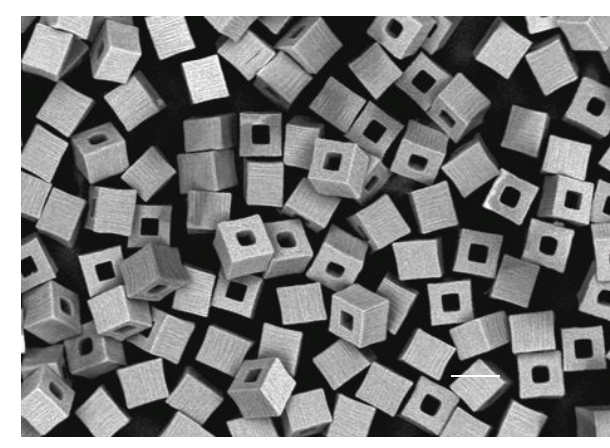

(a)

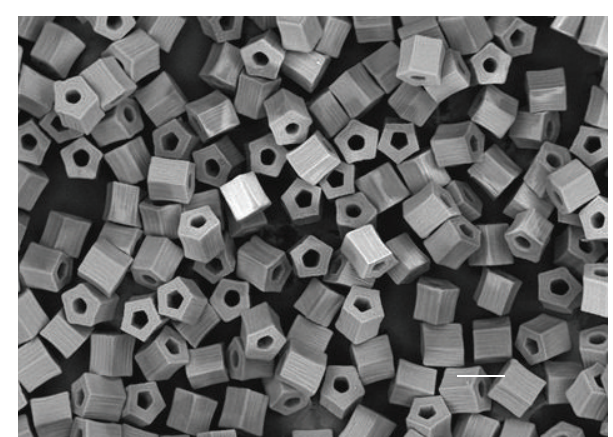

(b)

FIGURE 5: SEM micrographs of $\mathrm{SiO}_{2}$ single via particles in square and pentagonal cross sections. Scale bar $=100 \mu \mathrm{m}$.

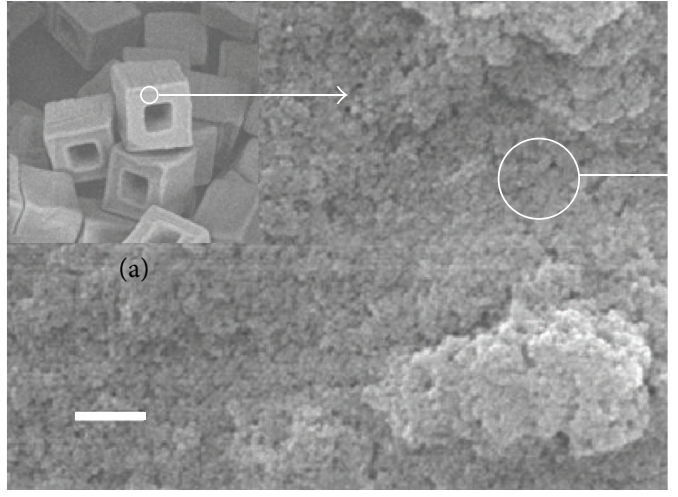

(b)

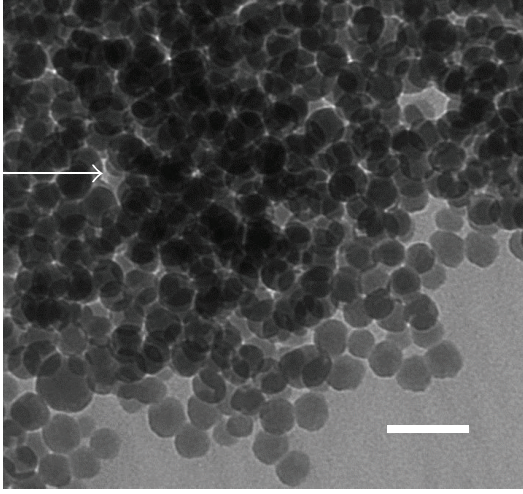

(c)

Figure 6: (a) Low mag. SEM micrograph of $\mathrm{SiO}_{2}$ particles. (b) Higher mag. SEM of the $\mathrm{SiO}_{2}$ particle surface. $\mathrm{Scale}$ bar $=200 \mathrm{~nm}$. (c) TEM of the as received Ludox particles. Scale bar $=50 \mathrm{~nm}$.

highly porous $\mathrm{Al}_{2} \mathrm{O}_{3}$ microparticles. It might also explain why the theoretical prediction of volume reduction is over $95 \%$, when only approximately $\sim 60 \%$ reduction is measured experimentally.

Figure 8 shows multivia PEG-DA/SiO ${ }_{2}$ composite particles. The multiplicity of vias in the particles is a means to create particles with ordered porosity as well as providing additional surface area.

The data for BET surface area, pore volume, and pore size collected by nitrogen physisorption are shown in Table 2 . Prior to data collection, standard microporous and mesoporous reference materials were evaluated to confirm the accuracy of the instrument. The inorganic, single via, $\mathrm{SiO}_{2}$ microparticles in square, circular, and pentagonal cross sections recorded BET surface area values in the range of 56$69 \mathrm{~m}^{2} / \mathrm{g}$ with pore volume and pore sizes of $0.08-0.09 \mathrm{~cm}^{3} / \mathrm{g}$ and $1.9-2.8 \mathrm{~nm}$, respectively. Additionally, the $\mathrm{SiO}_{2}$ particle with four vias yielded a BET surface area, pore volume, and pore size of $114 \mathrm{~m}^{2} / \mathrm{g}, 0.15 \mathrm{~cm}^{3} / \mathrm{g}$, and $2.1 \mathrm{~nm}$, respectively. Similarly, the $\mathrm{Al}_{2} \mathrm{O}_{3}$ via particles recorded specific surface area values ranging from 73 to $81 \mathrm{~m}^{2} / \mathrm{g}$.

The BET data collected for the single and multivia particles has several implications. First, the presence of the vias in these particles makes them superior with respect to surface area for physisorption compared with analogous solid particles. Second, based on the data obtained thus far, it is not obvious that the particle cross-sectional shapes influenced the BET values. Finally, for fully densified solid microparticles, one would expect surface area measurements less than $0.1 \mathrm{~m}^{2} / \mathrm{g}$. Thus, obtaining surface areas from 50 to $80 \mathrm{~m}^{2} / \mathrm{g}$, prior to optimization, suggests that the particles have potential for applications where structure and high surface area are important. In order to optimize the particles for future applications, removal of templates and volatile fractions by washing and calcination could increase the particle BET surface area by more than one order of magnitude $[43,44]$. Also, control of the binder concentration could be another method to improve the porosity.

\section{Conclusions}

The SFL method is used to synthesize two-dimensionally extruded, nonspherical PEG-DA/SiO ${ }_{2}$ and PEG-DA/ $\mathrm{Al}_{2} \mathrm{O}_{3}$ composite particles from which the corresponding inorganic particles are obtained by heat treatment. The approach represents an indirect application of the SFL technique to obtain 


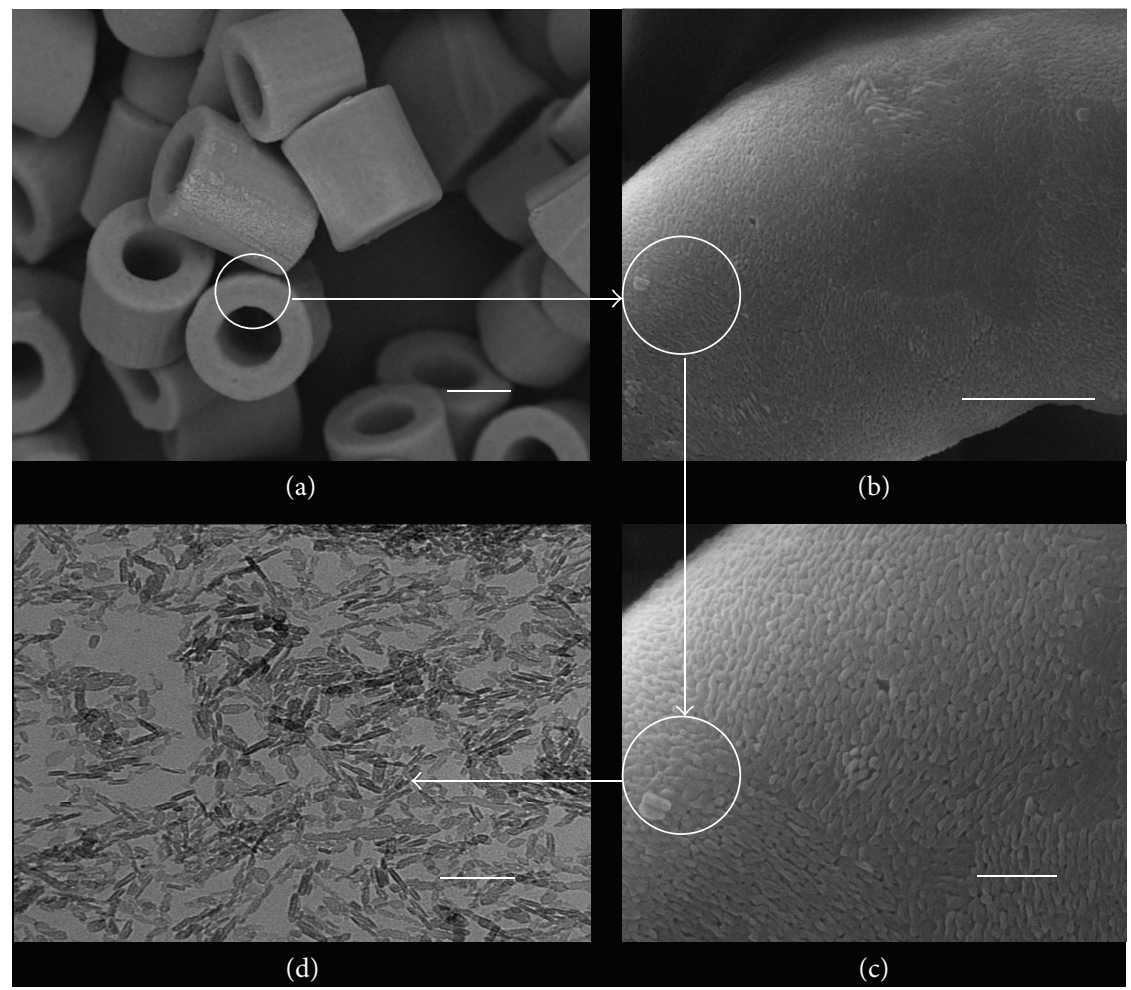

Figure 7: SEM micrographs of single via $\mathrm{Al}_{2} \mathrm{O}_{3}$ microparticles with a circular cross section (a). (b) and (c) are successively higher magnification images to reveal the microstructural features of (a). (d) TEM of the $\mathrm{Al}_{2} \mathrm{O}_{3}$ nanoparticles in suspension as received. Scale bars: (a) $=50 \mu \mathrm{m},(\mathrm{b})=10 \mu \mathrm{m},(\mathrm{c})=100 \mathrm{~nm}$, and (d) $=100 \mathrm{~nm}$.

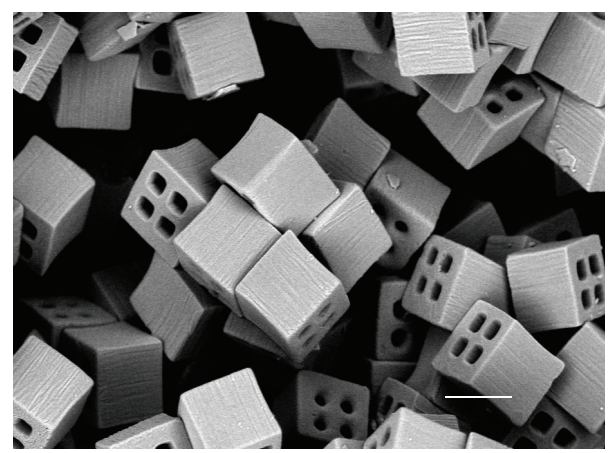

FIGURE 8: SEM micrograph of multivia PEG-DA/SiO 2 composite microparticles. Scale bars: $100 \mu \mathrm{m}$.

high fidelity, consolidated inorganic particles. Microparticles of $\mathrm{SiO}_{2}$ and $\mathrm{Al}_{2} \mathrm{O}_{3}$ with square, circular, and pentagonal cross-sectional shapes with vias have been synthesized. Two objective lens magnifications of $10 \mathrm{x}$ and $20 \mathrm{x}$ were used to demonstrate the reduction in particle cross-sectional dimensions whereas two different channel depths $(60-80 \mu \mathrm{m}$ and 220-250 $\mu \mathrm{m}$ ) controlled the particle axial dimension. High magnification SEM revealed that the $\mathrm{SiO}_{2}$ microparticles are formed from compacted spherical $\mathrm{SiO}_{2}$ nanoparticles. However, the high magnification image for $\mathrm{Al}_{2} \mathrm{O}_{3}$ revealed an array of rod-like $\mathrm{Al}_{2} \mathrm{O}_{3}$ nanoparticles interspersed with
TABLE 2: BET surface area of single via $\mathrm{SiO}_{2}$ and $\mathrm{Al}_{2} \mathrm{O}_{3}$ particles of different cross sections and multi-via cubic $\mathrm{SiO}_{2}$ particles.

(a) $\mathrm{SiO}_{2}$

\begin{tabular}{lccc}
\hline $\begin{array}{l}\text { Particle Cross } \\
\text { Section Shape }\end{array}$ & $\begin{array}{c}\text { Specific Surface } \\
\text { Area } \\
\left(\mathrm{m}^{2} / \mathrm{g}\right)\end{array}$ & $\begin{array}{c}\text { Pore Volume } \\
\left(\mathrm{cm}^{3} / \mathrm{g}\right)\end{array}$ & $\begin{array}{c}\text { Pore Size } \\
(\mathrm{nm})\end{array}$ \\
\hline Square & 56 and $114^{*}$ & $\begin{array}{c}0.09 \text { and } \\
0.15^{*}\end{array}$ & $\begin{array}{c}1.9 \text { and } \\
2.1^{*}\end{array}$ \\
Pentagonal & 69 & 0.08 & 1.2 \\
Circular & 64 & 0.08 & 2.8 \\
\hline $\begin{array}{l}\text { Range of particle } \\
\text { dimension }(\mu \mathrm{m})\end{array}$ & & $40-47$ & \\
\hline
\end{tabular}

(b) $\mathrm{Al}_{2} \mathrm{O}_{3}$

\begin{tabular}{lccc}
\hline $\begin{array}{l}\text { Particle Cross } \\
\text { Section Shape }\end{array}$ & $\begin{array}{c}\text { Specific Surface } \\
\text { Area } \\
\left(\mathrm{m}^{2} / \mathrm{g}\right)\end{array}$ & $\begin{array}{c}\text { Pore Volume } \\
\left(\mathrm{cm}^{3} / \mathrm{g}\right)\end{array}$ & $\begin{array}{c}\text { Pore Size } \\
(\mathrm{nm})\end{array}$ \\
\hline Square & 73 & 0.45 & 3.5 \\
Circular & 81 & 0.73 & 2.9 \\
\hline $\begin{array}{l}\text { Range of particle } \\
\text { dimension }(\mu \mathrm{m})\end{array}$ & & $75-90$ & \\
\hline
\end{tabular}

${ }^{*} 4$ via particle.

microvoids. BET surface area measurements for the single via inorganic particles were $56-69 \mathrm{~m}^{2} / \mathrm{g}$ for the $\mathrm{SiO}_{2}$ particles 
and $73-81 \mathrm{~m}^{2} / \mathrm{g}$ for the $\mathrm{Al}_{2} \mathrm{O}_{3}$ particles. Additionally, multivia cubic $\mathrm{SiO}_{2}$ particles measured $114 \mathrm{~m}^{2} / \mathrm{g}$ in BET surface area as determined by nitrogen physisorption isotherms at liquid nitrogen temperature $(77 \mathrm{~K})$. The surface areas achieved suggest that the particles have great potential for applications in areas like catalysis and sieving where structure and surface area control are important.

\section{Conflict of Interests}

The authors declare that there is no conflict of interests regarding the publication of this paper.

\section{Acknowledgments}

This material is based upon work supported by the National Science Foundation under Grant no. DMR-0611612. Any opinions, findings, and conclusions or recommendations expressed in this material are those of the authors and do not necessarily reflect the views of the National Science Foundation. David Baah gratefully acknowledges a graduate fellowship from Alabama EPSCoR.

\section{References}

[1] J. P. Best, Y. Yan, and F. Caruso, "The role of particle geometry and mechanics in the biological domain," Advanced Healthcare Materials, vol. 1, no. 1, pp. 35-47, 2012.

[2] N. S. Oltra, J. Swift, A. Mahmud, K. Rajagopal, S. M. Loverde, and D. E. Discher, "Filomicelles in nanomedicine-from flexible, fragmentable, and ligand-targetable drug carrier designs to combination therapy for brain tumors," Journal of Materials Chemistry B, vol. 1, no. 39, pp. 5177-5185, 2013.

[3] L. Tao, W. Hu, Y. Liu, G. Huang, B. D. Sumer, and J. Gao, "Shapespecific polymeric nanomedicine: emerging opportunities and challenges," Experimental Biology and Medicine, vol. 236, no. 1, pp. 20-29, 2011.

[4] H. Zou, S. Wu, and J. Shen, "Polymer/silica nanocomposites: preparation, characterization, propertles, and applications," Chemical Reviews, vol. 108, no. 9, pp. 3893-3957, 2008.

[5] C. Burda, X. Chen, R. Narayanan, and M. A. El-Sayed, "Chemistry and properties of nanocrystals of different shapes," Chemical Reviews, vol. 105, no. 4, pp. 1025-1102, 2005.

[6] Y.-W. Jun, J.-S. Choi, and J. Cheon, "Shape control of semiconductor and metal oxide nanocrystals through nonhydrolytic colloidal routes," Angewandte Chemie, vol. 45, no. 21, pp. 34143439, 2006.

[7] A. Phuruangrat, P. Jitrou, P. Dumrongrojthanath et al., "Hydrothermal synthesis and characterization of $\mathrm{Bi}_{2} \mathrm{MoO}_{6}$ nanoplates and their photocatalytic activities," Journal of Nanomaterials, vol. 2013, Article ID 789705, 8 pages, 2013.

[8] A. R. Tao, S. Habas, and P. Yang, "Shape control of colloidal metal nanocrystals," Small, vol. 4, no. 3, pp. 310-325, 2008.

[9] Y. Xia, Y. Xiong, B. Lim, and S. E. Skrabalak, "Shape-controlled synthesis of metal nanocrystals: simple chemistry meets complex physics?" Angewandte Chemie, vol. 48, no. 1, pp. 60-103, 2009.

[10] I. A. Rahman and V. Padavettan, "Synthesis of Silica nanoparticles by Sol-Gel: size-dependent properties, surface modification, and applications in silica-polymer nanocomposites-a review," Journal of Nanomaterials, vol. 2012, Article ID 132424, 15 pages, 2012.

[11] J. Hu, M. Chen, X. Fang, and L. Wu, "Fabrication and application of inorganic hollow spheres," Chemical Society Reviews, vol. 40, no. 11, pp. 5472-5491, 2011.

[12] X. W. Lou, L. A. Archer, and Z. Yang, "Hollow micro-/nanostructures: synthesis and applications," Advanced Materials, vol. 20, no. 21, pp. 3987-4019, 2008.

[13] A.-H. Lu, E. L. Salabas, and F. Schüth, "Magnetic nanoparticles: synthesis, protection, functionalization, and application," Angewandte Chemie International Edition, vol. 46, no. 8, pp. 12221244, 2007.

[14] J.-H. Lee, "Gas sensors using hierarchical and hollow oxide nanostructures: overview," Sensors and Actuators B: Chemical, vol. 140, no. 1, pp. 319-336, 2009.

[15] X. W. Lou, Y. Wang, C. Yuan, J. Y. Lee, and L. A. Archer, “Template-free synthesis of $\mathrm{SnO}_{2}$ hollow nanostructures with high lithium storage capacity," Advanced Materials, vol. 18, no. 17, pp. 2325-2329, 2006.

[16] X. W. Lou, C. Yuan, and L. A. Archer, "Shell-by-shell synthesis of tin oxide hollow colloids with nanoarchitectured walls: cavity size tuning and functionalization," Small, vol. 3, no. 2, pp. 261265, 2007.

[17] X. W. D. Lou, L. A. Archer, and Z. Yang, "Hollow micro-/nanostructures: synthesis and applications," Advanced Materials, vol. 20, no. 21, pp. 3987-4019, 2008.

[18] G. Wang, T. Liu, X. Xie, Z. Ren, J. Bai, and H. Wang, "Structure and electrochemical performance of $\mathrm{Fe}_{3} \mathrm{O}_{4}$ /graphene nanocomposite as anode material for lithium-ion batteries," Materials Chemistry and Physics, vol. 128, no. 3, pp. 336-340, 2011.

[19] Y. Wang, T. J. Merkel, K. Chen, C. A. Fromen, D. E. Betts, and J. M. Desimone, "Generation of a library of particles having controlled sizes and shapes via the mechanical elongation of master templates," Langmuir, vol. 27, no. 2, pp. 524-528, 2011.

[20] Q. Zhang, B. Lin, and J. Qin, "Synthesis of shape-controlled particles based on synergistic effect of geometry confinement, double emulsion template, and polymerization quenching," Microfluidics and Nanofluidics, vol. 12, no. 1-4, pp. 33-39, 2012.

[21] Q. Zhang, W. Wang, J. Goebl, and Y. Yin, "Self-templated synthesis of hollow nanostructures," Nano Today, vol. 4, no. 6, pp. 494-507, 2009.

[22] Y. Zhao and L. Jiang, "Hollow micro/nanomaterials with multilevel interior structures," Advanced Materials, vol. 21, no. 36, pp. 3621-3638, 2009.

[23] F. Caruso, R. A. Caruso, and H. Möhwald, "Nanoengineering of inorganic and hybrid hollow spheres by colloidal templating," Science, vol. 282, no. 5391, pp. 1111-1114, 1998.

[24] V. Giridharan, Y. Yun, P. Hajdu et al., "Microfluidic platforms for evaluation of nanobiomaterials: a review," Journal of Nanomaterials, vol. 2012, Article ID 789841, 14 pages, 2012.

[25] X. Wang, S. Li, L. Wang et al., "Microfluidic device for controllable chemical release via field-actuated membrane incorporating nanoparticles," Journal of Nanomaterials, vol. 2013, Article ID 864584, 6 pages, 2013.

[26] C.-X. Zhao, L. He, S. Z. Qiao, and A. P. J. Middelberg, "Nanoparticle synthesis in microreactors," Chemical Engineering Science, vol. 66, no. 7, pp. 1463-1479, 2011.

[27] D. Baah and T. Floyd-Smith, "Microfluidics for particle synthesis from photocrosslinkable materials," Microfluidics and Nanofluidics, vol. 17, no. 3, pp. 431-455, 2014. 
[28] S.-M. Yang, S.-H. Kim, J.-M. Lim, and G.-R. Yi, "Synthesis and assembly of structured colloidal particles," Journal of Materials Chemistry, vol. 18, no. 19, pp. 2177-2190, 2008.

[29] J. Lee, P. W. Bisso, R. L. Srinivas, J. J. Kim, A. J. Swiston, and P. S. Doyle, "Universal process-inert encoding architecture for polymer microparticles," Nature Materials, vol. 13, no. 5, pp. 524-529, 2014.

[30] H. An, H. B. Eral, L. Chen, M. B. Chen, and P. Doyle, "Synthesis of colloidal microgels using oxygen-controlled flow lithography," Soft Matter, vol. 10, no. 38, pp. 7595-7605, 2014.

[31] D. Baah, J. Tigner, K. Bean, N. Walker, B. Britton, and T. FloydSmith, "Microfluidic synthesis and post processing of nonspherical polymeric microparticles," Microfluidics and Nanofluidics, vol. 12, no. 1-4, pp. 657-662, 2012.

[32] S. C. Chapin, D. C. Pregibon, and P. S. Doyle, "High-throughput flow alignment of barcoded hydrogel microparticles," Lab on a Chip-Miniaturisation for Chemistry and Biology, vol. 9, no. 21, pp. 3100-3109, 2009.

[33] D. Dendukuri, S. S. Gu, D. C. Pregibon, T. A. Hatton, and P. S. Doyle, "Stop-flow lithography in a microfluidic device," Lab on a Chip, vol. 7, no. 7, pp. 818-828, 2007.

[34] D. Baah, T. Donnell, J. Tigner, and T. Floyd-Smith, "Stop flow lithography synthesis of non-spherical metal oxide particles," Particuology, vol. 14, pp. 91-97, 2014.

[35] R. F. Shepherd, J. C. Conrad, T. Sabuwala, G. G. Gioia, and J. A. Lewis, "Structural evolution of cuboidal granular media," Soft Matter, vol. 8, no. 17, pp. 4795-4801, 2012.

[36] R. F. Shepherd, P. Panda, Z. Bao et al., "Stop-flow lithography of colloidal, glass, and silicon microcomponents," Advanced Materials, vol. 20, no. 24, pp. 4734-4739, 2008.

[37] J.-L. Blin, A. Léonard, Z.-Y. Yuan et al., "Hierarchically mesoporous/macroporous metal oxides templated from polyethylene oxide surfactant assemblies," Angewandte Chemie, vol. 42, no. 25, pp. 2872-2875, 2003.

[38] K. Du, X. Cui, and B. Tang, "Template-directed synthesis of hollow silica beads by an interfacial sol-gel route," Chemical Engineering Science, vol. 98, pp. 212-217, 2013.

[39] K. W. Bong, S. C. Chapin, D. C. Pregibon, D. Baah, T. M. FloydSmith, and P. S. Doyle, "Compressed-air flow control system," Lab on a Chip-Miniaturisation for Chemistry and Biology, vol. 11, no. 4, pp. 743-747, 2011.

[40] D. K. Hwang, J. Oakey, M. Toner et al., "Stop-Flow lithography for the production of shape-evolving degradable microgel particles," Journal of the American Chemical Society, vol. 131, no. 12, pp. 4499-4504, 2009.

[41] F. Iskandar, A. B. D. Nandiyanto, K. M. Yun, C. J. Hogan Jr., K. Okuyama, and P. Biswas, "Enhanced photocatalytic performance of brookite $\mathrm{TiO}_{2}$ macroporous particles prepared by spray drying with colloidal templating," Advanced Materials, vol. 19, no. 10, pp. 1408-1412, 2007.

[42] J.-F. Chen, H.-M. Ding, J.-X. Wang, and L. Shao, "Preparation and characterization of porous hollow silica nanoparticles for drug delivery application," Biomaterials, vol. 25, no. 4, pp. 723727, 2004.

[43] S. H. Kim, B. Y. H. Liu, and M. R. Zachariah, "Ultrahigh surface area nanoporous silica particles via an aero-sol-gel process," Langmuir, vol. 20, no. 7, pp. 2523-2526, 2004.

[44] Q. Liu, P. Deshong, and M. R. Zachariah, "One-step synthesis of dye-incorporated porous silica particles," Journal of Nanoparticle Research, vol. 14, no. 7, article 923, 2012. 

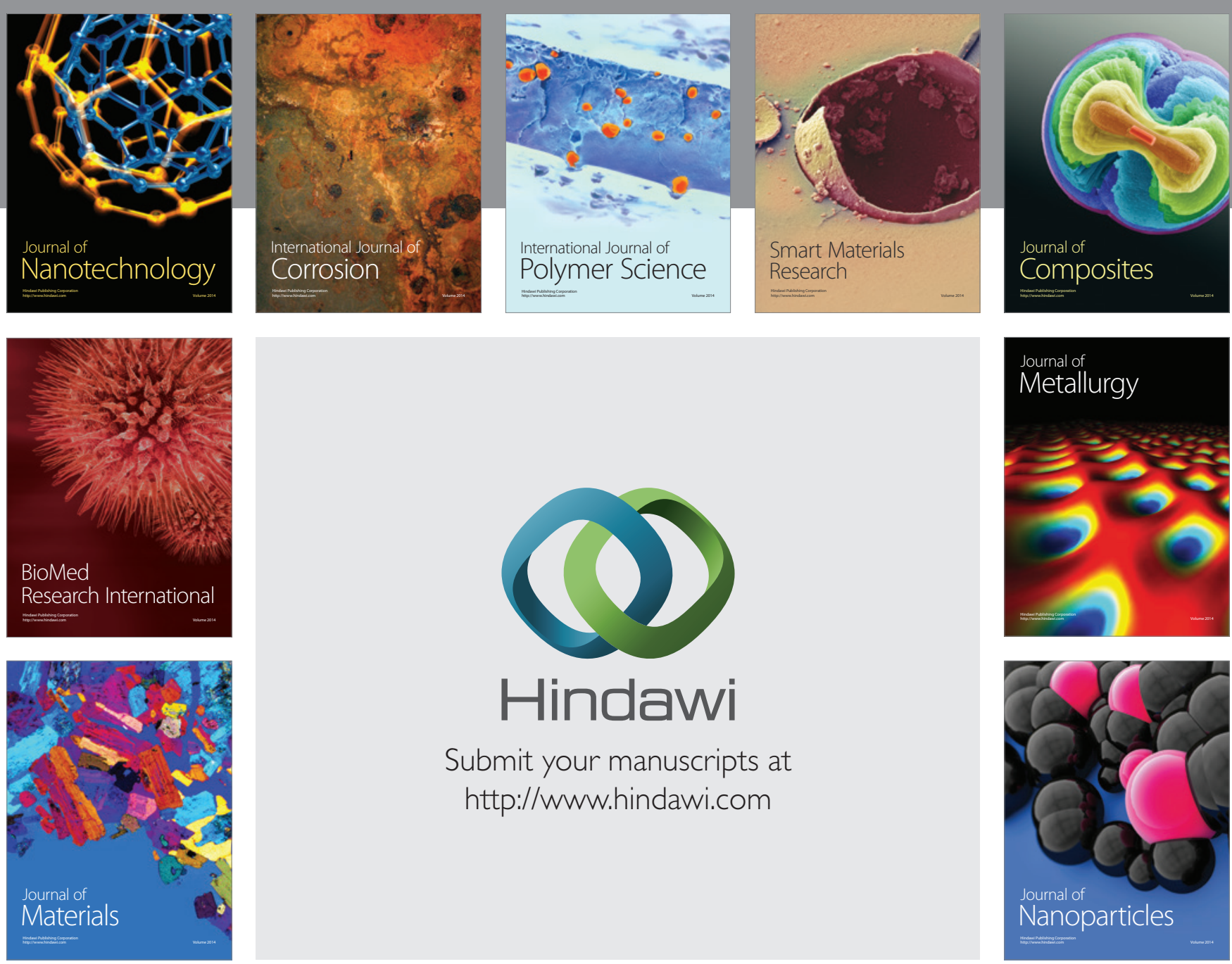

Submit your manuscripts at http://www.hindawi.com
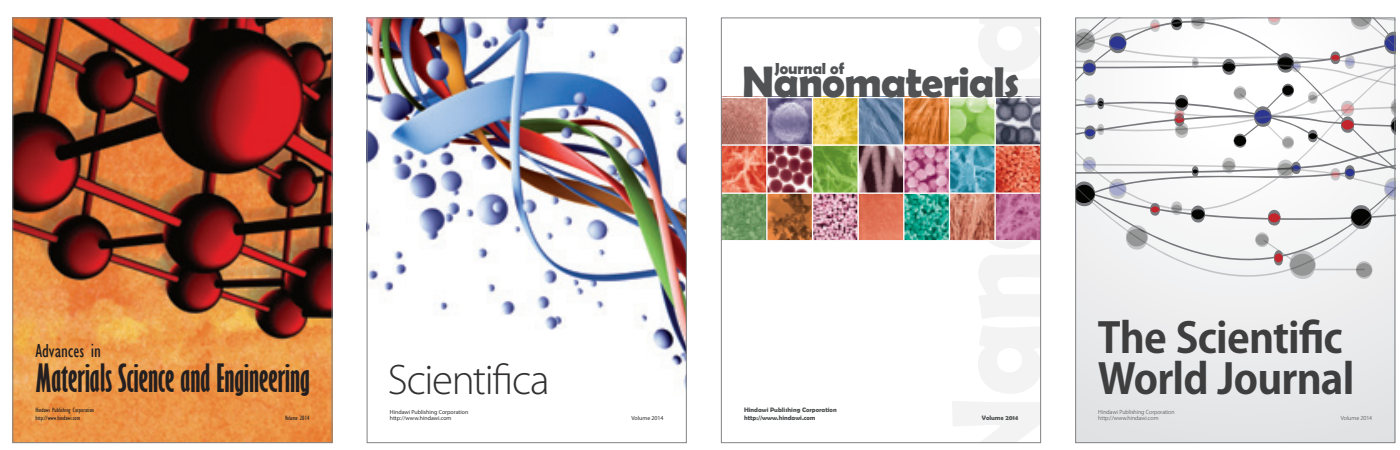

\section{The Scientific World Journal}
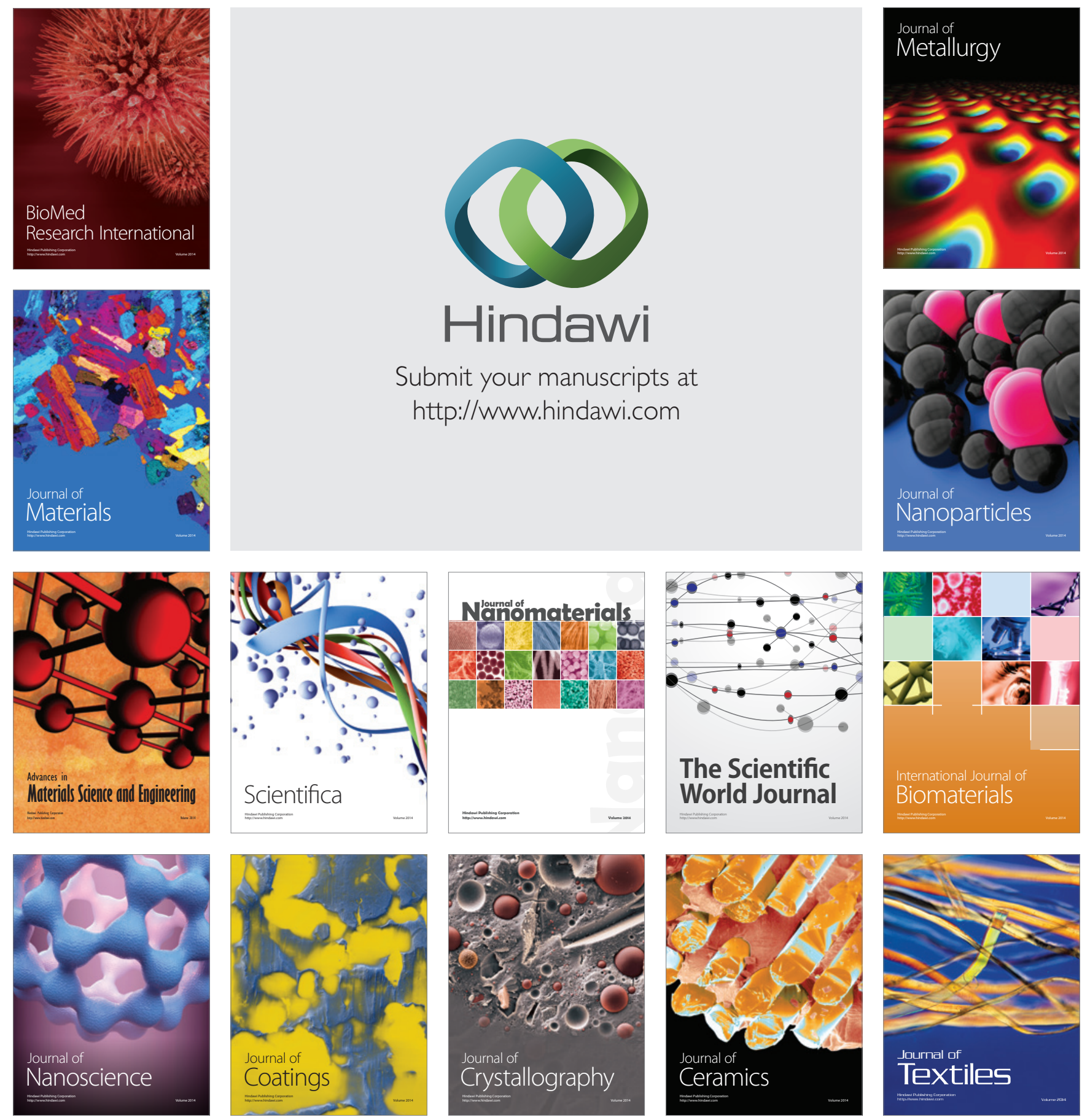